\title{
Genome-wide transcriptome profiling provides new insights into flower bud dormancy in pear
}

\author{
L.L. SUN ${ }^{1}$, R. ZHANG ${ }^{1}$, S.T. HE ${ }^{2}$, J.P. LIU' ${ }^{1}$, and G.Q. LIU ${ }^{3 *}$ \\ College of Agriculture, Guizhou University, Guizhou, 550025 Guiyang, P.R. China ${ }^{1}$ \\ College of Forestry, Guizhou University, Guizhou, 550025 Guiyang, P.R. China ${ }^{2}$ \\ College of Tobacco Science, Guizhou University, Guizhou, 550025 Guiyang, P.R. China ${ }^{3}$
}

\begin{abstract}
Dormancy is important for the pear (Pyrus pyrifolia) to survive a harsh environment. The molecular base of dormancy in pear, especially in some local cultivars, is still unclear. Genome-wide transcriptome analysis in flower buds of cv. Huangli (an excellent local cultivar native to Guizhou mountain area in China) was conducted to explore the mechanism regulating bud dormancy in pear. For the release of endo-dormancy 223 chilling hours (CHs) was needed in Huangli flower buds, which was less than in commercial cultivars. Comparisons of transcript amounts among seven dates during dormancy (30 Oct. vs. 15 Nov., 15 Nov. vs. 30 Nov., 30 Nov. vs. 15 Dec., 15 Dec. vs. 30 Dec., 30 Dec. vs. 15 Jan., and 15 Jan vs. $15 \mathrm{Feb}$.), resulted in the detection of $1064,1057,541,412,577$, and 3814 differentially expressed transcripts, respectively. The reference genome of pear was used to align the RNA-Seq reads and to measure the transcript expression. Gene ontology $(G O)$ and Kyoto encyclopedia of genes and genomes $(K E G G)$ were then used to annotate the transcript descriptions and to assign a pathway to each transcript. The results revealed, that secondary metabolite biosynthesis, especially phenylpropanoid biosynthesis pathway, was the most enriched pathway out of 132 pathways. These interesting results indicated that dormancy in Huangli might be regulated mainly by secondary metabolite biosynthesis pathway, and the two continuous dormant stages (endo-dormancy and eco-dormancy) might be regulated specifically by phenylpropanoid biosynthesis and plant hormone signal transduction, respectively.
\end{abstract}

Additional key words: chilling hours, phenylpropanoid biosynthesis pathway, Pyrus pyrifolia, RNA-Seq.

\section{Introduction}

Pear (Pyrus pyrifolia) is one of the most widely grown fruit trees and China is the leading pear-producing country (Zhao et al. 2018). Similar to other deciduous fruit trees, pear enters a dormant period during winter that provides an adaptive capacity to resist a low temperature, which affects growth and production during the next season. Recent reports have shown the impact of global warming on fruit trees, which negatively impact chilling accumulation and bud dormancy release (Luedeling et al. 2011), leading to irregular flowering and decreased fruit production. An important strategy to cope with this problem and ensure sustainable fruit production is using new pear cultivars with the low chilling requirement. The local cultivar Huangli, native to Guizhou Karst mountain area, has low chilling requirement, which makes it a prospective cultivar for the global warming future. However, the underlying molecular mechanism regulating flower bud dormancy in this cultivar is not clear. Therefore, it is important to explore the transcriptome associated with dormancy (Campoy et al. 2011).

The overwintering dormancy in perennial deciduous plants is a very complicated process. Traditional studies explored and cloned a single gene regulated dormancy in the buds (Jiménez et al. 2009, Mathiason et al. 2009, Sasaki et al. 2011, Liu et al. 2012, Bai et al. 2013, Zhu et al. 2015). It may be a synergistic mechanism involving multiple genes rather than the result of single genes. In recent years, the focus has been on a comprehensive and systematic approach to understanding the function and expression of genes involved in regulating dormancy. Researchers

Submitted 18 April 2019, last revision 27 August 2019, accepted 13 September 2019.

Abbreviations: CH - chilling hour; DEGs - differentially expressed genes; EST - expressed sequence tag; NGS - next-generation sequencing; SSH - suppression subtractive hybridization; GO - gene ontology; KEGG - Kyoto encyclopedia of genes and genomes. Acknowledgements: We acknowledge funding from the National Natural Science Foundation of China (31560537) and the Guizhou Provincial Natural Science Foundation (J [2015]2040).

* Corresponding author; e-mail: liuguoqin75@126.com. 
have applied suppression subtractive hybridization (SSH) and cDNA microarray technologies to study the molecular regulation of dormancy. Mazzitelli et al. (2007) adopted cDNA microarray technology to screen 327 expressed sequence tags (ESTs) related to hibernation in raspberry. Yamane et al. (2006) applied SSH technology to screen MADS-box genes relevant to hibernation in plum. Mathiason et al. (2009) applied SSH technology to discover over 130 differentially expressed genes (DEGs) related to substances and energy metabolism and signal transduction and cell growth during the low-temperature cumulative process in Vitis riparia . Leida et al. (2010) adopted SSH to validate 101 genes specifically expressed during bud dormancy release in peach. Santamaría et al. (2011) discovered a multitude of differentially expressed genes related to dormancy and growth by building SSH libraries of dormant and germinating buds of chestnut.

Recently, with the advantages of high accuracy, high throughput, high sensitivity, etc., RNA-Seq technology can detect gene expression in the scope of the whole genome. Gai et al. (2012) and Habu et al. (2012) applied 454 high-throughput sequencing platforms for transcriptome analysis during dormancy in peony and plum. Liu et al. (2012) and Bai et al. (2013) applied RNA-Seq technology for transcriptome analysis in pear cvs. Suli and Kosui during the conversion of endodormancy and ecodormancy. Next-generation sequencing (NGS) has been used to investigate the physiological and molecular mechanisms of bud dormancy in fruit trees and other crops (Liu et al. 2012, Ueno et al. 2013, Zhu et al. 2015). However, no attempts have yet been made to study flower bud dormancy in some excellent local pear cultivars at the transcriptome level.

The present research was conducted to investigate the potential molecular mechanisms regulating flower bud dormancy in cv. Huangli. Using pear genome ( $\mathrm{Wu}$ et al. 2013) as the reference genome, RNA-Seq libraries of 7 periods during dormancy were constructed and in flower buds expression patterns of the differential genes were screened to understand the molecular mechanisms underlying the transcriptional control of overwintering bud dormancy.

\section{Materials and methods}

Plants and cultivation: Fifteen-year-old pear (Pyrus pyrifolia Nakai cv. Huangli) grafted on $P$. pashia rootstock was cultivated in the orchard (Weining Yi, Hui, and Miao Autonomous County, Guizhou, China). Current season shoots were collected at different dormancy stages from the same trees. The bud samples were collected on 30 October, 15 November, 30 November, 15 December, and 30 December in 2015, 15 January and 15 February in 2016. They were frozen in liquid nitrogen, and then stored at $-80{ }^{\circ} \mathrm{C}$ before extracting RNA. The buds were collected from shoots in three biological replicates at each stage and used an independent pool. The temperature was recorded using an automatic temperature and humidity recorder from October 2015 to March 2016. Accumulated chilling hours $(\mathrm{CH})$ were calculated using $7.2{ }^{\circ} \mathrm{C}$ model (Weinberger et al. 1950). The number of chilling hours at time $t$ was expressed as:

$$
C H t=\sum_{i=1}^{t} T_{7.2}, \text { with } T_{7.2}=\left[\begin{array}{ll}
\mathrm{T}<7.2^{\circ} \mathrm{C} & : 1 \\
\text { else } & : 0
\end{array}\right]
$$

$T$ - temperature, $t$ - time, $\mathrm{CH} t$ - number of chilling hours at time $\mathrm{t}$.

Dormancy status of flower buds was estimated as described previously (Liu et al. 2012). Twelve shoots (approximately $15 \mathrm{~cm}$ long, bearing 10 - 12 lateral flower buds were collected to measure the percentage of bud break. The shoots were incubated in a phytotron at a day/night temperature of $25 / 18^{\circ} \mathrm{C}$, a relative humidity of $75 \%$, and a 12-h photoperiod with an irradiance of $320 \mu \mathrm{mol} \mathrm{m} \mathrm{m}^{-2} \mathrm{~s}^{-1}$. The water was renewed and the ends of the shoots submerged in water were cut every $2-3 \mathrm{~d}$. The percentage of bud break was measured after $21 \mathrm{~d}$. Buds were considered breaking when a green leaf tip was visible at the end of the bud. Flower buds were considered at the endodormant status when the percentage of bud break was less than $50 \%$ and ecodormant status when the percentage of bud break was more than $50 \%$ (Niu et al. 2015).

Preparation of RNA-sequencing library, mRNA sequencing, and data processing: Total RNA was extracted as described previously (Liu et al. 2012). Nanodrop spectrophotometer was used to quantify the total RNA, and denaturing formaldehyde-containing agarose gel and Agilent 2100 (Agilent Technologies, Santa Clara, CA, USA) bioanalyzer was used to check the RNA integrity. The purified mRNA from each sample was used to prepare the RNA-Seq library, and sequencing was done using BGISEQ-500 by Beijing Genome Institute (BGI; Shenzhen, China). Twenty-one RNA-Seq libraries were made using RNA extracted from buds collected on seven different dates during dormancy. Each library was constructed using RNA from three biological replicates of each stage. The raw reads were filtered for removing low-quality sequences by SOAPnuke. The sequences from the digital gene expression profiling analysis were deposited in the NCBI sequence read archive (accession PRJNA557023). Clean reads were mapped to the reference genome (http: //peargenome. njau. edu. cn: 8004/default. asp? $\mathrm{d}=4 \& \mathrm{~m}=2$ ) (Wu et al. 2013) using HISAT. The gene expressions were calculated in terms of FPKM (fragments per kilobase of exon per million fragments mapped) by RSEM. The differentially expressed genes (DEGs) with diverge probability $\geq 0.8$ and $\log 2$ fold change $\geq 2$ were identified using NOISeq method. The DEGs identified were further subjected to gene ontology $(G O)$ and Kyoto encyclopedia of genes and genomes $(K E G G)$ enrichment analysis. The enriched $G O$ terms and $K E G G$ pathways with $P \leq 0.05$ were considered significantly enriched. 


\section{Results}

Only in few days daily maximum temperature was lower than $5^{\circ} \mathrm{C}$ and the period was discontinuous. However, the periods with daily minimum temperature lower than $5^{\circ} \mathrm{C}$ often occurred from October 2015 to February 2016. From Dec 2015 to Feb 2016, the daily mean temperature was lower than $5^{\circ} \mathrm{C}$ (Fig. 1 Suppl.).

The accumulated chilling hours increased gradually at intervals of $15 \mathrm{~d}$, increased gradually with time, from $30^{\text {th }}$ Oct 2015 to $30^{\text {th }}$ Dec 2015. The accumulated chilling hours $(\mathrm{CH})$ amounted to $626 \mathrm{CH}$ from $15^{\text {th }}$ Oct 2015 to $15^{\text {th }}$ Dec 2015 and added up to $912 \mathrm{CH}$ on $30^{\text {th }} \mathrm{Dec} 2015$. The accumulated chilling hours further amounted to $1734 \mathrm{CH}$ on $15^{\text {th }}$ Feb of the next year (2016) (Table 2 Suppl.).

To identify the DEGs associated with dormancy, the dormancy status of pear flower buds were first detected using excised shoots. We observed less than $50 \%$ of the buds broken in shoots sampled on Oct. 30, more than $50 \%$ of the buds broken on 15 Nov. and after then. Flower buds sampled on 30 Oct. were in endodormant status, while those collected on 15 Nov., 30 Nov., 15 Dec., 30 Dec. 15 Jan., and 15 Feb. were in ecodormant status (Fig. 1).

The RNA-Seq was used to detect the changes in gene expression on seven different dates (six comparisons) during bud dormancy in pear. Twenty-one libraries with three biological replicates for each date were sequenced to generate more than 24 million clean reads $(99.91 \%$ of the generated data) per library after eliminating low-quality reads and adaptor sequences from the raw reads. Q20 value was 95.98 - $97.04 \%$. HISAT (http://ccb.jhu.edu/software/ hisat/index.shtml) was used to map the clean reads to the reference genome of pear (Wu et al. 2013). The genome mapping ratio was $78.70-82.21 \%$. Sequencing data met the quality standards required for further analysis (Table 1 Suppl.). The heat map of correlations between samples showed that the correlation coefficients within the three biological replicates were higher than 0.97 .

The differential expression of transcripts was analyzed among seven different dates during pear flower

Table 1. The number of differently expressed genes belonging to different functional categories (BP - biological processes, CC - cellular components, MF - moleculat functions).

\begin{tabular}{lcccrr}
\hline & BP & CC & MF & Total & Ratio [\%] \\
\hline 30 Oct. vs. 15 Nov. & $742(13.65 \%)$ & $506(11.43 \%)$ & $442(13.10 \%)$ & 1690 & 12.77 \\
15 Nov. vs. 30 Nov. & $733(13.49 \%)$ & $609(43.26 \%)$ & $482(14.29 \%)$ & 1824 & 25.34 \\
30 Nov. vs. 15 Dec. & $317(5.83 \%)$ & $329(7.43 \%)$ & $217(6.43 \%)$ & 863 & 6.52 \\
15 Dec. vs. 30 Dec. & $292(5.37 \%)$ & $201(4.54 \%)$ & $184(5.46 \%)$ & 677 & 5.12 \\
30 Dec. vs. 15 Jan. & $362(6.66 \%)$ & $364(8.22 \%)$ & $256(7.59 \%)$ & 982 & 13.64 \\
15 Jan. vs. 15 Feb. & $2988(54.99 \%)$ & $2419(54.63 \%)$ & $1792(53.13 \%)$ & 7199 & 54.39 \\
Total & 5434 & 4428 & 3373 & 13235 & 100 \\
Ratio [\%] & 41.06 & 33.46 & 25.49 & 100 &
\end{tabular}

Table 2. Major Kyoto encyclopedia of genes and genomes $(K E G G)$ pathways in flower buds during winter. DEGs - differently expressed genes.

\begin{tabular}{llr}
\hline Groups & Pathway & Number of DEGs \\
\hline & biosynthesis of secondary metabolites & 161 \\
30 Oct. vs. 15 Nov. & phenylpropanoid biosynthesis & 44 \\
15 Nov. vs. 30 Nov. & starch and sucrose metabolism & 38 \\
& biosynthesis of secondary metabolites & 170 \\
& ribosome & 45 \\
30 Nov. vs. 15 Dec. & phenylpropanoid biosynthesis & 44 \\
& biosynthesis of secondary metabolites & 75 \\
15 Dec. vs. 30 Dec. & ribosome & 32 \\
& phenylpropanoid biosynthesis & 19 \\
30 Dec. $v s .15$ Jan. & biosynthesis of secondary metabolites & 91 \\
& phenylpropanoid biosynthesis & 23 \\
15 Jan. $v s .15$ Feb. & plant-pathogen interaction & 22 \\
& biosynthesis of secondary metabolites & 52 \\
& ribosome & 33 \\
& starch and sucrose metabolism & 19 \\
& biosynthesis of secondary metabolites & 420 \\
& ribosome & 164 \\
& plant hormone signal transduction & 119 \\
\hline
\end{tabular}


bud dormancy by pairwise comparison (Fig. 2). The analysis revealed that the greatest differences were examined in 15 Jan. vs. 15 Feb. comparison (1 683 downregulated transcripts and 2131 up-regulated transcripts) followed by 30 Oct. vs. 15 Nov., 15 Nov. vs. 30 Nov., 30 Dec. vs. 15 Jan., 30 Nov. vs. 15 Dec., and 15 Dec. $v s$. 30 Dec. The almost same number of genes were significantly up-regulated and down-regulated in period 30 Nov. vs. 15 Dec.

Cluster analysis divided the transcripts into six groups (30 Oct. vs. 15 Nov., 15 Nov. vs. 30 Nov., 30 Nov. vs. 15

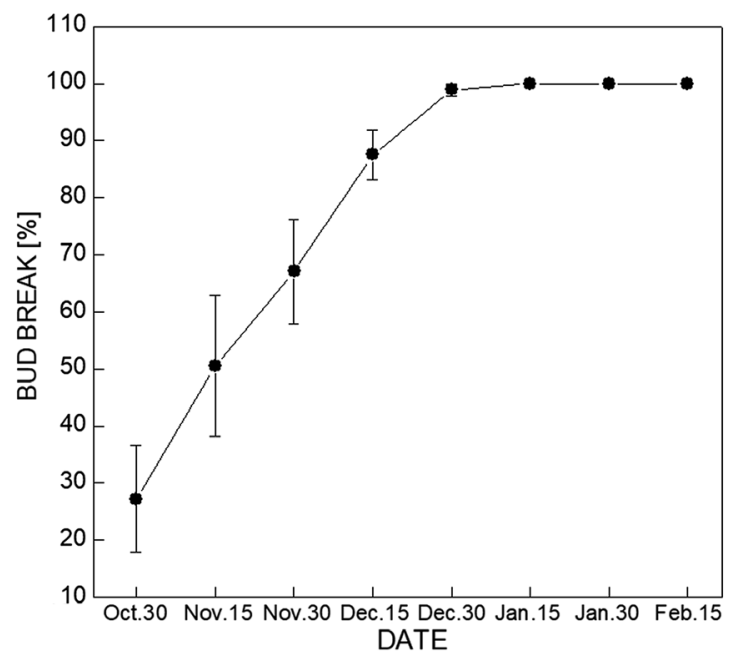

Fig. 1. Dormancy status of pear flower buds during winter. Shoots were collected and incubated in a phytotron to measure the percentage of bud break and so to estimate the dormancy status of flower buds from 2015 to 2016. The percentage of bud break was measured after $21 \mathrm{~d}$. Means \pm SEs, $n=3$.

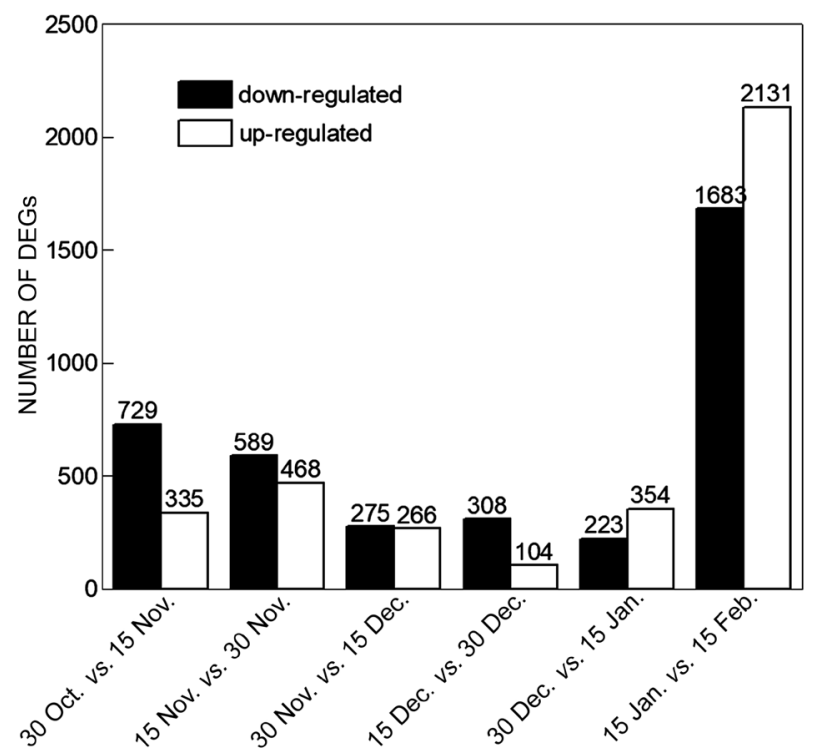

Fig. 2. Changes in the number of differentially expressed genes (DEGs) among different comparisons during dormancy. The DEGs with diverge probability $\geq 0.8$ and $\log 2$ fold change $\geq 2$ were identified, and the numbers of up-regulated and downregulated genes in comparison of different libraries are shown side-by-side.
Dec., 15 Dec. vs. 30 Dec., 30 Dec. vs. 15 Jan., and 15 Jan. 15 vs. 15 Feb.). Analysis revealed that 335 (31.5\%), 468 (44.28\%), 266 (49.17\%), 104 (25.24\%), 354 (61.35\%), and $2131(55.87 \%)$ transcripts were up-regulated and 729 (68.5\%), 589 (55.72\%), 275 (50.83\%), 308 (74.76 $\%), 223(38.65 \%)$, and $1683(44.13 \%)$ transcripts were down-regulated in groups 30 Oct. vs. 15 Nov., 15 Nov. vs. 30 Nov., 30 Nov. vs. 15 Dec., 15 Dec. vs. 30 Dec., 30 Dec. vs. 15 Jan., and 15 Jan. 15 vs. 15 Feb., respectively. In 30 Oct. vs. 15 Nov., 15 Nov. vs. 30 Nov., and 30 Nov. vs. 15 Dec. 15 groups, majority of transcripts were downregulated; while in the other three groups majority of transcripts were up-regulated (Fig. 3).

The GO analysis showed that the biological functions of DEGs focused on three main categories including biological process (BP), cellular component (CC), and molecular function (MF) (Table 1). BP was the highly represented category $(5434 ; 41.06 \%)$ followed by CC (4 428; $33.46 \%$ ), and MF (3 373; $25.49 \%)$. The 30 Oct. vs. 15 Nov., 15 Nov. vs. 30 Nov., 30 Nov. vs. 15 Dec., 15 Dec. vs. 30 Dec., 30 Dec. vs. 15 Jan., and 15 Jan. vs. 15 Feb. comparisons represented 1690 (12.77\%), 1824 (13.78\%), $863(6.52 \%), 677$ (5.12\%), $982(7.42 \%)$, and $7199(54.39 \%)$ transcripts, respectively, of the total 13235 transcripts annotated into major GO categories.

Transcripts associated with the BP subcategories including metabolic process $(198,27.01 \%$; 229, $94.32 \%$; 112 , $35.33 \%$; 97, 33.22\%; 125, 34.53\%; 877, $29.35 \%)$ and cellular process $(146,19.68 \% ; 129,17.60 \% ; 56$, $17.67 \%$; 60, $20.55 \%$; 80, $22.10 \%$; 648, $21.69 \%$ ) were recognized in 30 Oct. vs. 15 Nov., 15 Nov. vs. 30 Nov., 30 Nov. vs. 15 Dec., 15 Dec. vs. 30 Dec., 30 Dec. vs. 15 Jan., and 15 Jan. vs. 15 Feb. comparisons. Transcripts associated with the $\mathrm{CC}$ subcategories such as cell (121, $23.91 \%$; 140, $22.99 \%$; 80, $24.32 \%$; 47, $23.38 \%$; 94,

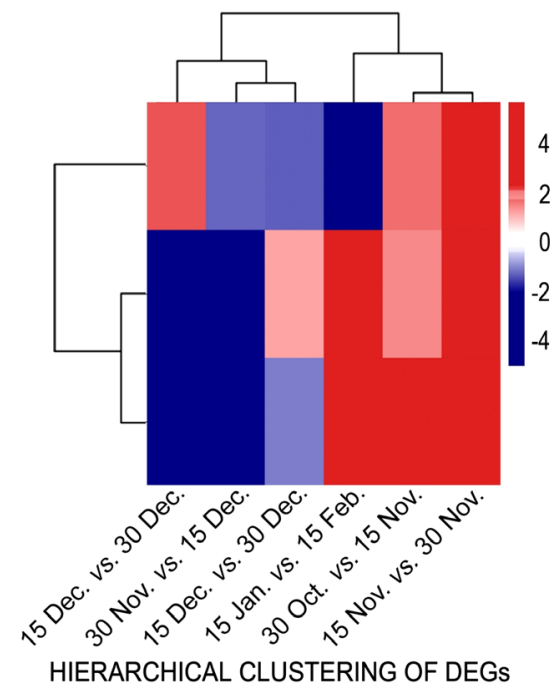

Fig. 3. Cluster analysis of gene expressions based on log ratio reads per kilobase per million mapped reads data. The clusters display expression patterns for a subset of differently expressed genes in six comparisons. Each column represents an experimental condition, and each row represents a gene. Red means up-regulated and blue means down-regulated. 
$25.82 \% ; 558,23.07 \%)$ and cell parts $(121,23.91 \% ; 140$, $22.99 \%$; 80, $24.32 \%$; 47, $23.38 \%$; 94, $25.82 \%$; 558, $23.07 \%$ ) were identified in 30 Oct. vs. 15 Nov., 15 Nov. vs. 30 Nov., 30 Nov. vs. 15 Dec., 15 Dec. vs. 30 Dec., 30 Dec. vs. 15 Jan., and 15 Jan. vs. 15 Feb. comparisons. Transcripts associated with the MF subcategories such as catalytic activity $(221,50 \% ; 218,45.23 \% ; 81,37.33 \%$; 93, $50.54 \% ; 92,35.94 \% ; 800,44.64 \%$ ), and binding (184, 41.63\%; 190, 39.42\%; 94, $43.32 \%$; 72, $39.13 \%$; $114,44.53 \% ; 759,42.35 \%$ ) were recognized in 30 Oct. vs. 15 Nov., 15 Nov. vs. 30 Nov., 30 Nov. vs. 15 Dec., 15 Dec. vs. 30 Dec., 30 Dec. vs. 15 Jan., and 15 Jan. vs. 15 Feb. comparisons.

During $K E G G$ enrichment classification of DEGs, 8399 DEGs were allocated to 132 pathways, and the most enriched pathway was secondary metabolite biosynthesis (969 transcripts) followed by the ribosome (300 transcripts) and phenylpropanoid biosynthesis (217 transcripts) at seven different dormancy dates. Secondary metabolite biosynthesis was the most significantly regulated pathway in all six comparisons, and phenylpropanoid biosynthesis was the most significantly regulated secondary metabolite biosynthesis pathway in four comparisons (30 Oct. vs. 15 Nov., 15 Nov. vs. 30 Nov., 30 Nov. vs. 15 Dec., and
15 Dec. vs. 30 Dec. 30) (Table 2).

In the current study, 969 transcripts linked to secondary metabolite biosynthesis were identified for six groups. A total of 131 DEGs were annotated in phenylpropanoid biosynthesis of which 35 were up-regulated and 96 were down-regulated during all seven dates of dormancy.

In the 30 Oct. vs. 15 Nov. comparison, 45 DEGs were involved in phenylpropanoid biosynthesis, with 10 up-regulated and 35 down-regulated. The upregulated genes were mainly annotated anthocyanidin 3-O-glucosyltransferase, while the down-regulated genes were mainly annotated GDSL esterase/lipase. In the 15 Nov. vs. 30 Nov. comparison, 44 DEGs were involved in phenylpropanoid biosynthesis with 16 up-regulated and 28 down-regulated. The up-regulated genes were mainly annotated peroxidase, while the down-regulated genes were mainly annotated GDSL esterase/lipase and peroxidase. In the 30 Nov. vs. 15 Dec. comparison, 19 DEGs were involved in phenylpropanoid biosynthesis with 8 up-regulated and 11 down-regulated. The upregulated genes were mainly annotated caffeic acid 3-O-methyltransferase 1-like and neurofilament medium polypeptide, while the down-regulated genes were mainly annotated GDSL esterase/lipase and peroxidase. In the
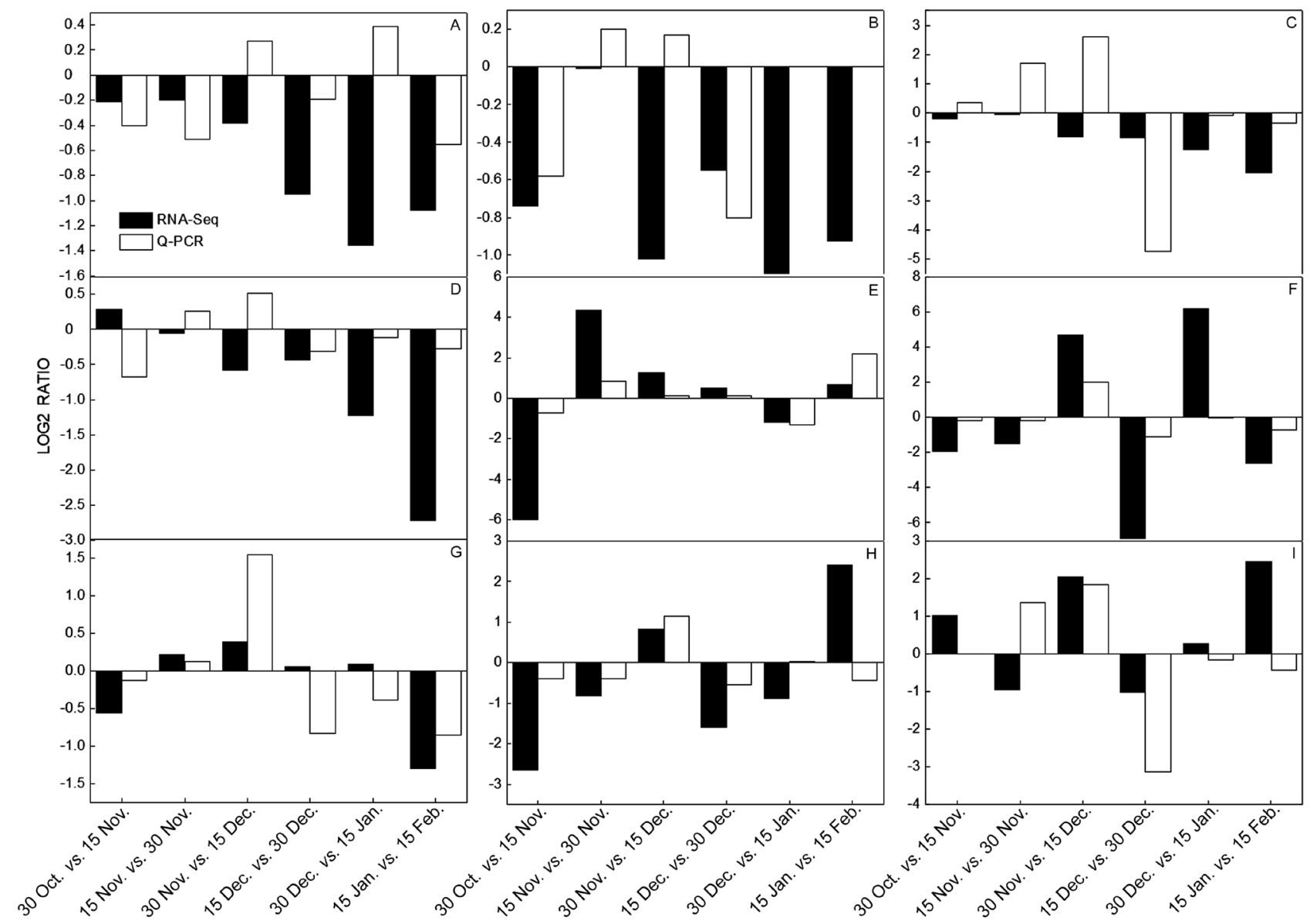

Fig. 4. Verification of relative expressions of nine differently expressed genes $(A$ - Pbr019338.1; $B$ - Pbr019339.1; $C$ - Pbr019340.1; $D$ - Pbr038022.1; $E$ - Pbr007773.1; F - Pbr003716.1; $G$ - Pbr007701.1; H - Pbr000689.1; $I$ - Pbr013897.1) by reverse transcription quantitative PCR. 
15 Dec. vs. 30 Dec. comparison, 23 DEGs were involved in phenylpropanoid biosynthesis with 1 up-regulated and 22 down-regulated. The up-regulated genes were annotated vinorine synthase-like, while the down-regulated genes were mainly annotated caffeic acid 3-O-methyltransferase and peroxidase GDSL esterase/lipase.

Nine DEGs were chosen for reverse transcription quantitative PCR analysis to verify the precision and reproducibility of the transcriptome analysis results. In each case, most of the reverse transcription quantitative PCR results closely related to transcript level assessment by the RNA-sequencing analysis (Fig. 4).

\section{Discussion}

Dormancy is a complex phenomenon that enables perennial plants to survive in winter. Low temperature has been proven to be critical in the dormancy of pear (Heide and Prestrud 2005, Olsen 2006). However, inadequate winter chilling due to global warming has influenced many fruit trees including pears and resulted in delayed autumn and spring phenologies (Cannell and Smith et al. 1982, Young 1992, Luedeling et al. 2009, 2011, Anderson et al. 2010, Campoy et al. 2011). Therefore, it is important to investigate the molecular mechanism of chilling mediated dormancy in pear.

In recent years, the focus has shifted from exploration and cloning of a single gene to comprehensive systematic study of expression and function of more genes. With the advantages of high accuracy, high throughput, high sensitivity, etc., RNA-Seq technology can detect gene expression in the scope of the whole genome. Liu et al. (2012) and Bai et al. (2013) have conducted RNA-Seq, focus on the transcriptome analysis of Pyrus pyrifolia cvs. Suli and Kosui during the conversion period of the endo-dormancy and eco-dormancy on the basis of no reference genome. In the current study, the genomewide transcriptomic analysis was performed using RNAsequencing approach in flower buds of'Pyrus pyrifolia cv. Huangli, an excellent local variety native to Guizhou mountain area in China, during the progression of dormancy under different chilling accumulation.

In this study, the release of endodormancy of Huangli flower buds was before Nov. 15, so the chilling requirement was less than $223 \mathrm{CH}$ (Fig. 1). This was quite less than for cvs. Suli and Kosui, which may be due to the differences in species and ecological conditions.

We found that the number of DEGs and their expression profiles were different on different collection dates. Although the total number of DEGs was close in the endodormant stage (30 Oct. vs. 15 Nov. and 15 Nov. vs. 30 Nov.), the number of significantly up-regulated DEGs was fewer than that of down-regulated DEGs. The number of genes significantly up-regulated and down-regulated was the closest during the transition from endodormancy to ecodormancy (30 Nov. vs. 15 Dec.). The total number of DEGs gradually increased during ecodormancy (15 Dec. vs. 30 Dec., 30 Dec. vs. 15 Jan., and 15 Jan. vs. 15 Feb.). The number of significantly upregulated
DEGs was less than that of downregulated DEGs at the early stage of ecodormancy (15 Dec. vs.30 Dec.), and the number of significantly upregulated DEGs was more than that of downregulated DEGs with the progression of ecodormancy. Hedley et al. (2010) found that the activity of gene was the lowest at the beginning of dormancy and peaked during bud break in blackcurrant. Liu et al. (2012) reported that the number of DEGs during endodormancy was less than that during ecodormancy, which increased with the release of endodormancy in pear cv. Suli.

The DEGs participated in several different $K E G G$ pathways during dormancy. Secondary metabolite biosynthesis was the most significantly regulated pathway in all six comparisons of which phenylpropanoid biosynthesis was the most significantly regulated for the first four comparisons and plant hormone signal transduction was the major pathway for the latter two comparisons (Table 2). These interesting results indicated that the whole process of dormancy in cv. Huangli was regulated mainly by secondary metabolite biosynthesis pathways, and endodormancy and ecodormancy were regulated by phenylpropanoid biosynthesis and plant hormone signal transduction, respectively. The downregulation of DEGs annotated GDSL esterase/lipase was the major pattern at the endodormant stage. These results were quite different from the previous findings (Ramos et al. 2005, Mazzitelli et al. 2007, Horvath 2009, Leida et al. 2010) and may provide meaningful and reliable information for understanding bud dormancy in pear.

In conclusion, the current work revealed the importance of secondary metabolite biosynthesis especially phenylpropanoid pathway in pear flower bud dormancy.

\section{References}

Anderson, J., Horvath, D., Chao, W., Foley, M.: Bud dormancy in perennial plants: a mechanism for survival. - In: Lubzens, E., Cerda, J., Clark, M.H. (ed.): Dormancy and Resistance in Harsh Environments. Pp. 69-90. Springer, Berlin 2010.

Bai, S., Saito, T., Sakamoto, D., Ito, A., Fujii, H., Moriguchi, T.: Transcriptome analysis of Japanese pear (Pyrus pyrifolia Nakai) flower buds transitioning through endodormancy. Plant Cell Physiol. 54: 1132-1151, 2013.

Campoy, J.A., Ruiz, D., Egea, J.: Dormancy in temperate fruit trees in a global warming context: a review. - Sci. Hort. 130: 357-372, 2011.

Cannell, M., Smith, R.: Climatic warming, spring budburst and forest damage on trees. - J. appl. Ecol. 23:177-191, 1986.

Gai, S., Zhang, Y., Mu, P., Liu, C., Liu, S., Dong, L., Zheng, G.: Transcriptome analysis of tree peony during chilling requirement fulfillment: assembling, annotation and markers discovering. - Gene 497: 256-262, 2012.

Habu, T., Yamane, H., Igarashi, K.: 454-pyrosequencing of the transcriptome in leaf and flower buds of Japanese apricot (Prunus mume Sieb. et Zucc.) at different dormant stages. Jap. Soc. hort. Sci. 81: 239-50, 2012.

Hedley, P.E., Russell., J.R., Jorgensen., L., Gordon., S., Morris., J.A., Hackett., C.A., Cardle., L., Brennan., R.: Candidate genes associated with bud dormancy release in blackcurrant (Ribes nigrum L.). - BMC Plant Biol. 10: 202, 2010.

Heide, O., Prestrud, A.: Low temperature, but not photoperiod, 
controls growth cessation and dormancy induction and release in apple and pear. - Tree Physiol. 25: 109-114, 2005.

Horvath, D.: Common mechanisms regulate flowering and dormancy. - Plant Sci. 177: 523-31, 2009.

Jiménez, S., Lawton-Rauh, A.L., Reighard, G.L., Abbott, A.G., Bielenberg, D.G.: Phylogenetic analysis and molecular evolution of the dormancy associated MADS-box genes from peach. - BMC Plant Biol. 9: 1, 2009.

Leida, C., Terol, J., Marti, G., Agusti, M., Llacer, G., Badenes, M.L., Rios, G.: Identification of genes associated with bud dormancy release in Prunus persica by suppression subtractive hybridization. - Tree Physiol. 30: 655-666, 2010.

Liu, G., Li, W., Zheng, P., Xu, T., Chen, L., Liu, D., Teng, Y.: Transcriptomic analysis of 'Suli' pear (Pyrus pyrifolia white pear group) buds during the dormancy by RNA-Seq. - BMC Genomics 13: 1, 2012.

Luedeling, E., Girvetz, E.H., Semenov, M.A., Brown, P.H.: Climate change affects winter chill for temperate fruit and nut trees. - PLoS ONE 6: e20155, 2011.

Luedeling, E., Zhang, M., Girvetz, E.H.: Climatic changes lead to declining winter chill for fruit and nut trees in California during 1950 - 2099. - PLoS ONE 4: e6166, 2009.

Mathiason, K., He, D., Grimplet, J., Venkateswari, J., Galbraith, D.W., Or, E., Fennell, A.: Transcript profiling in Vitis riparia during chilling requirement fulfillment reveals coordination of gene expression patterns with optimized bud break. - Funct. integr. Genomics 9: 81-96, 2009.

Mazzitelli, L., Hancock, R.D., Haupt, S., Walker, P.G., Pont, S.D.A., McNicol, J., Cardle, L., Morris, J., Viola, R., Brennan, R.: Co-ordinated gene expression during phases of dormancy release in raspberry (Rubus idaeus L.) buds. - J. exp. Bot. 58: 1035-1045, 2007.

Niu, Q., Li, J., Cai, D., Qian, M., Jia, H., Bai, S., Zheng, X.: Dormancy-associated MADS-box genes and microRNAs jointly control dormancy transition in pear (Pyrus pyrifolia white pear group) flower bud. - J. Exp. Bot. 67: 239-257, 2015.

Olsen, J.E.: Mechanisms of dormancy regulation. - Acta. Hort.
??: 727-730, 2006.

Ramos, A., Pérez-Solís, E., Ibáñez, C., Casado, R., Collada, C., Gómez, L. Allona, I.: Winter disruption of the circadian clock in chestnut. - Proc. nat. Acad. Sci. USA 102: 7037-7042, 2005.

Santamaria, M.E., Rodriguez, R., Canal, M.J., Toorop, P.E.: Transcriptome analysis of chestnut (Castanea sativa) tree buds suggests a putative role for epigenetic control of bud dormancy. - Ann Bot. 108: 485-498, 2011.

Sasaki, R., Yamane, H., Ooka, T., Jotatsu, H., Kitamura, Y., Akagi, T., Tao, R.: Functional and expressional analyses of PmDAM genes associated with endodormancy in Japanese apricot. - Plant Physiol. 157: 48-97, 2011.

Ueno, S., Klopp, C., Leplé, J.C., Derory, J., Noirot, C., Léger, V., Le, P.G. Transcriptional profiling of bud dormancy induction and release in oak by next-generation sequencing. - BMC Genomics 14: 236, 2013.

Weinberger, J.H. Chilling requirements of peach varieties. - J. amer. Soc. hort. Sci. 56: 122-128, 1950.

Wu, J., Wang, Z., Shi, Z., Zhang, S., Ming, R., Zhu, S. Chen, N.J.: The genome of the pear (Pyrus bretschneideri Rehd.). Genome Res. 23: 396-408, 2013.

Yamane, H., Kashiwa, Y., Kakehi, E., Yonemori, K., Mori, H., Hayashi, K., Iwamoto, K., Tao, R., Kataoka, I.: Differential expression of dehydrin in flower buds of two Japanese apricot cultivars requiring different chilling requirements for bud break. - Tree Physiol. 26:1559-1563, 2006.

Young, E.: Timing of high temperature influences chilling negation in dormant apple trees. - J. amer. Soc. hort. Sci. 117: 271-273, 1992.

Zhao, Y.Q., Tian, Y.L., Wang, L.M., Geng, G.M., Zhao, W.J., Hu, B.S., Zhao, Y.F. Fire blight disease, a fast-approaching threat to apple and pear production in China. - J. integr. Agr. 17: 60345-60347, 2018.

Zhu, Y., Li, Y., Xin, D., Chen, W., Shao, X., Wang, Y., Guo, W.: RNA-Seq-based transcriptome analysis of dormant flower buds of Chinese cherry (Prunus pseudocerasus). - Gene 555: 362-76, 2015. 